\title{
A Multicentric Study of Long-term Rhythm Patterns in Heart Rate
}

\author{
Rebeca Goya-Esteban ${ }^{1}$, Óscar Barquero-Pérez ${ }^{1}$, Javier Alzueta ${ }^{2}$, Xavier Viñolas ${ }^{3}$, Nuria Basterra ${ }^{4}$, \\ Enrique García $^{5}$, Julián Villacastín ${ }^{6}$, Ignacio Fernandez-Lozano ${ }^{7}$, Josep Brugada $^{8}$, \\ José B Martinéz-Ferrer ${ }^{9}$, Francisco Zumalde ${ }^{10}$, María Martín-Méndez ${ }^{11}$, Fernando Chavarría-Asso ${ }^{11}$, \\ Arcadi García-Alberola ${ }^{12}$, José Luis Rojo-Álvarez ${ }^{1}$ \\ ${ }^{1}$ Rey Juan Carlos University, Fuenlabrada, Madrid \\ 2 Virgen de la Victoria Hospital, Málaga, Spain \\ ${ }^{3}$ Santa Creu i Sant Pau Hospital, Barcelona, Spain \\ ${ }^{4}$ Navarra Hospital Center, Pamplona, Spain \\ ${ }^{5}$ Vigo University Hospital Center, Vigo, Spain \\ ${ }^{6}$ Clínico San Carlos Hospital, Madrid, Spain \\ ${ }^{7}$ Puerta de Hierro University Hospital, Madrid, Spain \\ ${ }^{8}$ Clinic Hospital, Barcelona, Spain \\ ${ }^{9}$ Araba University Hospital, Vitoria, Spain \\ ${ }^{10}$ Galdakao Usansolo Hospital, Usansolo, Spain \\ ${ }^{11}$ Medtronic, Madrid, Spain \\ 12 Virgen de la Arrixaca University Hospital, Murcia, Spain
}

\begin{abstract}
Heart rate (HR) shows oscillations with different periods as a result of the sympatho-vagal balance. The most studied ones are short-period variations and the circadian pattern. However, the existence of rhythms of longer periods has not been systematically studied.

The aim of this work is to study HR long-period rhythms in a multicentric database including 336 patients in sinus rhythm, with implanted cardioverter defibrillator. We used two methodological approaches: First a rhythmometric procedure to automatically select the statistically significant rhythms present in the signal; Second, the LASSO path approach to analyze the order of activation of the rhythms, representing the importance of each rhythm.

Most of the population showed a significant annual rhythm (78\% day/80\% night). Weekly and quarterly rhythms were also present (weekly $26 \% 126 \%$; quarterly $22 \% / 21 \%$ ). Monthly rhythm was rarely present. Most present rhythm combinations were annual plus weekly $(21 \% / 21 \%)$ and annual plus quarterly (19\%/18\%). The order of activation given by the LASSO path was in agreement with the multicomponent rhythmometric model in the $86 \% / 85 \%$ of the cases.

The unusual long monitoring period, and the high number of patients, represent an ideal scenario to robustly assess the existence of long-term rhythms.
\end{abstract}

\section{Introduction}

Physiological rhythms arise from stochastic, nonlinear biological mechanisms interacting with a fluctuating environment. Separation of dynamics due to intrinsic rather than extrinsic mechanisms is not possible. Several studies indicate rich dynamics with differences between normal individuals and patients. Therefore, disruption of the rhythmic processes beyond normal bounds or emergence of abnormal rhythms is associated with disease. A possible approach to study these dynamics is to analyze qualitative aspects of simplified mathematical models of physiological systems [1].

Most biological variables vary greatly along several time scales in health and disease [2]. Heart rate (HR) shows oscillations with different periods. The most studied ones are short-period variations (seconds, minutes) and the circadian pattern. However, there is little information about the existence of rhythms of longer periods and they have not been systematically studied. One reason is the difficulty to ensemble the appropriate dabatabases with a large number of subjects and sufficient monitoring periods.

A suitable hypothesis is that physiological mechanisms in healthy subjects are more adaptable to environmental changes than those in pathological subjects, and maybe with a progressive deterioration of this adaptability related to the severity of the pathological condition. Several stud- 
ies have shown blunted or altered circadian rhythms of different physiological variables [3-5].

The aim of this study is to assess the adaptation capabilities, the patients responses, to stimuli of longer time periods. To this end we assessed the long-term HR rhythms present in a multicentric database including 336 patients. The database was assembled from SCOOP platform, a repository conveying around 12000 intracardiac electrograms stored by implanted cardioverter defibrillators (ICDs). The analysis was performed by means of two methodological approaches: First a rhythmometric procedure to automatically select the statistically significant rhythms present in the signal; Second, the LASSO path approach to analyze the order of activation of the rhythms, representing the importance of each rhythm.

The structure of the paper is as follows. Section 2 describes the multicentric database and the data preprocessing. In Section 3, the two methodological approaches are presented. Section 4 depicts the results. Finally, the conclusions are outlined in Section 5.

\section{Dataset}

A multicentric database including 381 patients in sinus rhythm, was assembled from SCOOP platform. SCOOP is a Spanish platform developed by Medtronic Ibérica S.A., it represents a pioneer scientific repository system in the cloud involving 48 national hospitals and conveying around 12000 intracardiac electrograms stored by ICDs and subsequently labeled by a scientific committee of expert physicians.

Daily and nightly mean HR was extracted from each patient record for its analysis. The monitoring period varies between 382 and 2348 days. From this dataset, patients with more than $5 \%$ of atrial or ventricular pacing at any point during the monitoring period were discarded. Also, assuming that some of the subjects could have suffered atrial fibrillation (AF) episodes during the monitoring period, a signal preprocessing was designed trying to detect AF episodes. Namely, HR samples differing in more than $15 \%$ of the mean of the previous 5 days were discarded. In this case, if more than the $20 \%$ of the samples are discarded in one patient, the patient is discarded from the analysis. Finally 336 patients remained for the study.

\section{Methods}

Extensive and dense time series collected over several decades show that nearly all biological variables display some degree of more or less periodic behaviour. When statistical methods are involved in data analysis time-dependence must be taken into account. Hence, in many cases, is useful to look upon a measurement series as consisting on a deterministic part, which may have both rhythmic and arrhythmic systematic components, and a noise part [2]. We used a rhythmometric procedure based on a multicomponent COSINOR approach and a non-parametric statistic test to automatically select the statistically significant rhythms present in the HR signal. Furthermore, we used the LASSO path approach to analyze the order of activation of the rhythms, representing the importance of each rhythm.

\subsection{Rhythmometric Analysis}

A data sequence can be represented by a temporal regression model known as the cosinor model [2], defined by

$$
y_{n}=M+A_{0} \cos \left(2 \pi f_{0} t_{n}+\phi_{0}\right)+e_{n}, n=1, \ldots, N,
$$

where $M$ denotes the rhythm-adjusted mean or MESOR (midline estimating statistic of rhythm), $A_{0}$ the amplitude, $f_{0}$ the fundamental frequency, $\phi_{0}$ the acrophase (i.e., the lag from a defined reference time point to the crest time in the cosine curve fitted to the data), and $N$ the signal length. The random variable $e_{n}$ corresponds to the difference between the observed sample $y_{n}$ and the value provided by the estimated regression model $\hat{y}_{n}$. The least squares (LS) method is applied to determine the regression parameters.

Joint characterization of a set of rhythms is provided by a multiple components model [2], which extends the regression model in (1) to become

$$
y_{n}=M+\sum_{i} A_{i} \cos \left(2 \pi f_{i} t_{n}+\phi_{i}\right)+e_{n},
$$

Where $f_{i}, A_{i}$ and $\phi_{i}$ are the frequency, the amplitude and the acrophase corresponding to each considered rhythm.

In the present study, weekly, monthly, quarterly and annual rhythms are considered, since those where the rhythms of interest selected by the cardiologist.

The LS method is used to find the regression parameters in (2). Next, the rhythmometric method leans toward a bootstrap hypothesis test [6] to select the rhythm components with statistical relevance in (2). The MESOR component is used as the starting rhythmometric model. In order to construct the final model, the method iteratively adds to the previous model the sinusoidal component in (2) with the highest amplitude. The mean square error (MSE) between the signal and each model $\left(E_{k}\right)$ is computed to obtain the difference between the residuals of the two models $\left(\Delta E_{k}=E_{k-1}-E_{k}\right)$. The statistical relevance of each model versus the previous one is assessed by using a paired bootstrap hypothesis test. A number of $B$ random resamplings with replacement of residuals $E_{k}$ are made to obtain $\Delta E_{k}$ for each resampling ( $\left.B=2500\right)$. A suitable statistical hypothesis test is to contrast the null hypothesis that the models have the same unexplained variance 

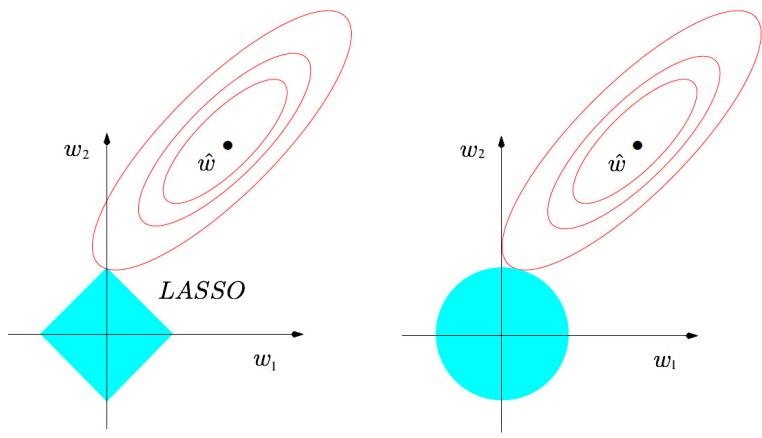

Figure 1. Comparison of estimation weights constraints between LASSO (left) and regularized regression (right). Adapted from [9].

$\left(\Delta E_{k}=0\right)$ against the alternative hypothesis that both models have different unexplained variance $\left(\Delta E_{k} \pm 0\right)$. The paired bootstrap hypothesis test determines that the addition of a new sinusoidal component is relevant when at least $97.5 \%$ of the $B$ values, for the estimated probability density function of $\Delta E_{k}$, are on the right-hand side of zero; a detailed explanation is found in [7].

\subsection{LASSO Path}

We propose, in this work, to use a differente approach to select rhythm components based on regularization techniques. As an alternative to the previous method, we can fit the complete rhythmometric model and constraint the coefficients associated to each component. We propose to use $L 1-$ norm penalization which has the effect of forcing some of the coefficient estimates to be exactly equal to zero, yielding to sparse models [8].

In order to use this approach, we need to reformulate the rhythmometric model as a linear one, rewriting 2 as

$$
y_{n}=M+\sum_{i} \alpha_{i} \cos \left(2 \pi f_{i} t_{n}\right)+\beta_{i} \sin \left(2 \pi f_{i} t_{n}\right)+e_{n},
$$

where $\alpha_{i}=A_{i} \cos \left(2 \pi \phi_{i}\right)$ and $\beta_{i}=-A_{i} \sin \left(2 \pi \phi_{i}\right)$. Collecting all the coefficients in a vector of weights $\boldsymbol{w}=\left[1, \alpha_{1}, \ldots, \alpha_{k}, \beta_{1}, \ldots, \beta_{k}\right]$, where $k$ is the number of rhythm components. Using a matrix $X$ to collect all the components and the MESOR, the rhythmometric model can be compactly written as

$$
\boldsymbol{y}=X \boldsymbol{w}+\boldsymbol{e}
$$

Weights of the model, $\boldsymbol{w}$, can be estimated using LS including a regularization term:

$$
\hat{\boldsymbol{w}}_{L_{2}}=\underset{\boldsymbol{w}}{\operatorname{argmin}}\|\boldsymbol{y}-X \boldsymbol{w}\|_{2}^{2}+\lambda\|\boldsymbol{w}\|_{2}^{2}
$$

The LASSO approach is shrinkage method like the previous one, but substituing $L_{2}$ norm in the regularization
Table 1. Percentage of patients showing each rhythm in the different time periods: Day, night and in both day and night.

\begin{tabular}{lccc}
\hline \hline Rhythm & Day (\%) & Night (\%) & Both (\%) \\
\hline Annual & 72 & 80 & 66 \\
Quarterly & 22 & 21 & 12 \\
Monthly & 3 & 3 & 1.5 \\
weekly & 26 & 26 & 12 \\
\hline \hline
\end{tabular}

penalty on weights by $L_{1}$ norm [9]:

$$
\hat{\boldsymbol{w}}_{L_{1}}=\underset{\boldsymbol{w}}{\operatorname{argmin}}\|\boldsymbol{y}-X \boldsymbol{w}\|_{2}^{2}+\lambda\|\boldsymbol{w}\|_{1}
$$

where $\|\boldsymbol{w}\|_{1}=\sum_{j=1}^{2 k+1}\left|w_{j}\right|$.

The nature of LASSO constraint allows to control the number of weights actives $\left(w_{i} \neq 0\right)$, so that, making $\lambda$ sufficiently large will cause some of the weights to be exactly zero, see Figure 1.

Linear models penalized with the $\mathrm{L}_{1}$ norm have sparse solutions, so that many of their estimated weights are zero. Accordingly, it is possible to use LASSO regression models to report the more important variables (features) of the model in the sense of mean squared error [10].

It is possible to perform a search on the regularization parameter $\lambda$. This is the so called LASSO path, in which starting at high values of $\lambda$ assures that all weights are equal to zero. Then we give smaller values for $\lambda$ up to zero. The idea is to register the event when a weight actives, that is, when $w_{i} \neq 0$, meaning that the associated rhythm component represent an important variable to explain the HR signal in the sense of minimum squared error [9]. We performed a complete LASSO path analysis in order to evaluate the activation dynamic of the rhythm components assuming that the first activated component represents the most relevant physiological rhythm.

\section{4. $\quad$ Results}

Table 1 shows that most of the patients showed a significant annual rhythm, either in the daily mean HR, or in the nightly mean HR, and in many cases in both time periods. Weekly and quarterly rhythms were also present, whereas monthly rhythm was rarely present. Most usual rhythm combinations were annual plus weekly and annual plus quarterly (see Tab. 2). Figure 2 shows an example of a HR signal and the fitted model where the annual and weekly rhythms were significant according to the procedure in Sec. 3.1.

The order of activation given by the LASSO path was in agreement with the rhythmometric model (Sec. 3.1) in the $86 \%$ day/85\% night of the patients. 
Table 2. Percentage of patients showing each rhythm combination in the different time periods.

\begin{tabular}{lccc}
\hline \hline Rhythm combination & Day (\%) & Night (\%) & Both (\%) \\
\hline Annual+weekly & 21 & 21 & 8 \\
Annual+Quarterly & 19 & 18 & 8 \\
Annual+Monthly & 1.5 & 2.7 & 1 \\
Ann.+Quart.+week. & 5 & 4 & 1 \\
\hline \hline
\end{tabular}

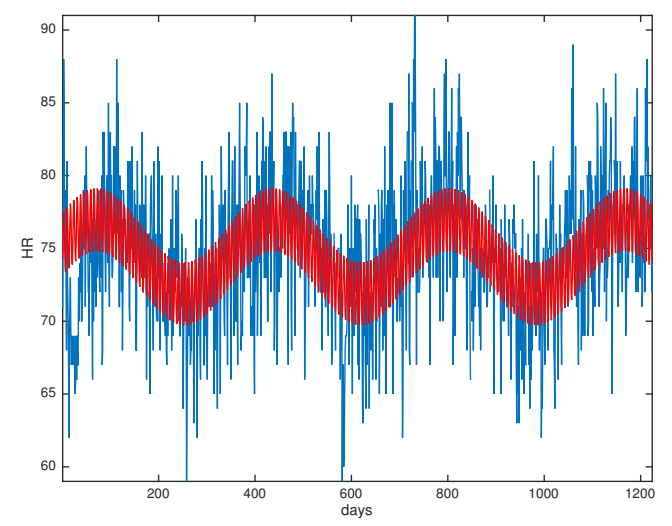

Figure 2. Example of mean HR evolution and fitted rhythmometric model. A combination of annual and weekly rhythms were significant for this signal.

\section{Conclusions}

The unusual long monitoring period, and the high number of patients in the database, represent an ideal scenario to robustly assess the existence of long-term rhythms.

In this study two technical approaches were in agreement in most cases, being the LASSO path used for the first time in rhythmometric analysis. The annual rhythm was by far the most significant one in the studied population.

Further studies will be devoted to assess the relation of the rhythm presence and the patient clinical data.

\section{Acknowledgements}

This work has been partially supported by the Research Projects from the Spanish Ministry of Economy and
Competitiveness, TEC2013-48439-C4-1-R and TEC201675161-C2-1-4.

\section{References}

[1] Glass L. Synchronization and rhythmic processes in physiology. Nature 2001;410(8):277-284.

[2] Bingham C, Arbogast B, Guillaume GC, Lee J, Halberg F. Inferential statistical methods for estimating and comparing cosinor parameters. Chronobiologia 1982;9(4):397-439.

[3] Guzzetti S, Dassi S, Pecis M, Casat R, Masu AM, Longoni P, Tinelli M, Cerutti S, Pagani M, Malliani A. Altered pattern of circadian neural control of heart period in mild hypertension. Journal of Hypertension 1991;9(9):831-838.

[4] Touitou Y, Bogdan A, Levi F, Benavides M, Auzeby A. Disruption of the circadian patterns of serum cortisol in breast and ovarian cancer patients: relationships with tumour marker antigens. British Journal of Cancer 1996; 74(8): 1248.

[5] Burger AJ, Charlamb M, Sherman HB. Circadian patterns of heart rate variability in normals, chronic stable angina and diabetes mellitus. International journal of cardiology 1999;71(1):41-48.

[6] Efron B, Tibshirani R. An introduction to the bootstrap. Chapman \& Hall, 1993.

[7] Goya-Esteban R, Mora-Jiménez I, Rojo-Alvarez JL, Barquero-Pérez O, Pastor-Pérez FJ, Manzano-Fernández S, Pascual-Figal DA, García-Alberola A. Heart rate variability on 7-day holter monitoring using a bootstrap rhythmometric procedure. IEEE Transactions on Biomedical Engineering 2010;57(6):1366-1376.

[8] James G, Witten D, Hastie T. An Introduction to Statistical Learning: With Applications in R. Springer, 2014.

[9] Tibshirani R. Regression shrinkage and selection via the lasso. Journal of the Royal Statistical Society Series B 1996;58(1):267-288

[10] Efron B, Hastie T, Johnstone I, Tibshirani R. Least angle regression. The Annals of Statistics 2004;32(2):407-499.

Address for correspondence:

Rebeca Goya-Esteban

Camino del Molino s/n, Departamental III D202, 28943, Fuenlabrada, Spain

rebeca.goyaesteban@urjc.es 